Original Research Article

\title{
A comparative study on efficacy of amitriptyline and escitalopram in the treatment of depression
}

\author{
Sushant Aryal ${ }^{1 *}$, Kajal Chakrabarti², Mayuri Gupta ${ }^{1}$
}

${ }^{1}$ Department of Pharmacology, Patan Academy of Health Sciences, Lalitpur, Nepal ${ }^{2}$ Department of Psychiatry, Nepal Medial College and Teaching Hospital Attarkhel, Jorpati, Kathmandu, Nepal

Received: 26 November 2017 Accepted: 28 December 2017

\section{*Correspondence to:}

Dr. Sushant Aryal,

Email: sushantaryal@ pahs.edu.np

Copyright: (C) the author(s), publisher and licensee Medip Academy. This is an openaccess article distributed under the terms of the Creative Commons Attribution NonCommercial License, which permits unrestricted noncommercial use, distribution, and reproduction in any medium, provided the original work is properly cited.

\begin{abstract}
Background: Several generations of antidepressant medication which act by distinct pharmacological mechanisms have been introduced for the treatment of depression; tricyclic antidepressants (TCAs) were first line of treatment for many years. However, over the last decade, selective serotonin reuptake inhibitors (SSRIs) have displaced TCAs, mainly because of better side effect profile. There are no references in literature on comparison of efficacy of TCAs and SSRIs in Nepalese population. This study attempted to compare the efficacy of amitriptyline, a reference standard TCA with escitalopram, a newer SSRI in Nepalese population.

Methods: An open level, randomised, prospective study was conducted for one year duration. Eighty outpatients suffering from major depression who met inclusion and exclusion criteria were randomly assigned to either amitriptyline or escitalopram group for four week study. Seventy one patients (amitriptyline N: 36, escitalopram N: 35) completed the study. Hamilton Depression Rating Scale (HDRS) was used to measure the antidepressant effect. Antidepressant efficacy was evaluated on reduction of HDRS score before and after therapy (End of four weeks).

Results: In amitriptyline group, mean percentage reduction in HDRS score was $58.29 \%$ (13.5 points), while in escitalopram group was $60.78 \%$ (14.03 points). Both the drugs significantly improved the HDRS score at the end of the study $(\mathrm{p}<0.05)$. On intergroup comparison, antidepressant efficacy of amitriptyline and escitalopram did not differ significantly from each other ( $p>0.05)$.

Conclusions: This study suggests that escitalopram is effective in the treatment of depression and its efficacy appears to be comparable to amitriptyline at the end of four weeks.
\end{abstract}

Keywords: Amitriptyline, Comparative, Escitalopram, Efficacy

\section{INTRODUCTION}

Depression is widely prevalent chronic recurrent psychiatric condition. ${ }^{1,2}$ It is a major public health problem worldwide which is associated with significant morbidity and mortality. ${ }^{1,2}$ Antidepressant (AD) medications play an important role in the management of depression. Tricyclic antidepressants (TCAs) formed mainstay of treatment for many decades. ${ }^{3}$ TCAs act primarily as a serotonin (5HT)- noradrenaline (NA) reuptake inhibitor. ${ }^{4,5}$ Amitriptyline is most widely used TCA and it is considered one of the reference standard against which new ADs are compared with respect to both efficacy and tolerability.

Many groups of AD drugs are now available, but it is the SSRIs, which have replaced TCAs as the first-choice drugs in the treatment of depression. ${ }^{3}$ SSRIs act primarily by selectively inhibiting the reuptake of $5 \mathrm{HT}$ with no effect 
on reuptake of NA. ${ }^{5,8,9}$ Escitalopram is the S-enantiomer of the racemic SSRI, citalopram, and is the most selective of the SSRIs studied to date. ${ }^{8,10,11}$

The AD efficacy of SSRIs has been well documented, but the comparative study has shown superior efficacy of escitalopram to conventional SSRIs. ${ }^{6,10-13}$ Comparative clinical trials indicate that SSRIs are as effective as TCAs, but relative safety and better acceptability of SSRIs has been the reason to their clinical success over TCAs. ${ }^{13-18}$

However, published data evaluating and comparing the efficacy of different ADs are lacking in Nepal. This study was designed to compare the efficacy of escitalopram, most selective SSRIs with amitriptyline, a reference standard drug in the treatment of depression.

\section{METHODS}

This study was conducted in Nepal Medical College and Teaching hospital (NMCTH), Jorpati, Kathmandu, Nepal for one year duration.

\section{Inclusion criteria}

- All the outpatients who were diagnosed as a case of major depression according to the Tenth Revision of International Classification of Disease-Diagnostic criteria for research with a minimum total score of 15 on HDRS at screening.

\section{Exclusion criteria}

- $\quad$ Pregnant or nursing women

- Women of childbearing age not using appropriate birth control methods.
- Acute or unstable medical problems as well as psychological diseases.

- History of substance abuse.

All the patients/guardian of patients was made to sign an informed consent form before their inclusion in the study.

A total of 80 patients were selected as research subjects. Subjects were then randomized equally into group 1 and group 2. Odd number patients were included in group 1 and they received amitriptyline, and even number patients in group 2 received escitalopram. Assessment of patient was done at screening, end of two week and end of four week for the efficacy. The collected data was entered in Statistical Package for Social Sciences (SPSS) 17 for analysis. Descriptive statistics was used to analyse sociodemographic data and $t$ test was applied to find the $\mathrm{p}$ value.

\section{RESULTS}

Out of the eighty patients enrolled in the study nine patients dropped out. Only seventy one patients, thirty six in the amitriptyline group and thirty five in escitalopram group were under observation throughout the period of the study.

\section{Socio-demographic profile}

Out of 71 patients, $31(43.7 \%)$ were male and $40(56.3 \%)$ were female. The mean age of the patients was $32.84 \pm 12.35$ years (range 17-64 years). The most commonly involved age group was 16-35 years (53 patients). A gender wise and age wise distribution of patients in two drug groups are summarized in Table 1.

Table 1: Age and gender wise distribution of patients in different drug groups $(n=71)$.

\begin{tabular}{|c|c|c|c|c|c|c|}
\hline \multirow{3}{*}{$\begin{array}{l}\text { Variable } \\
\text { Gender }\end{array}$} & \multicolumn{6}{|c|}{ Drug groups } \\
\hline & \multicolumn{2}{|c|}{ Amitriptyline $(n=36)$} & \multicolumn{2}{|c|}{ Escitalopram $(n=35)$} & \multicolumn{2}{|c|}{ Total $(n=71)$} \\
\hline & $\mathbf{n}$ & $\%$ & n & $\%$ & $\mathbf{n}$ & $\%$ \\
\hline Male & 16 & 44.4 & 15 & 42.9 & 31 & 43.7 \\
\hline Female & 20 & 55.6 & 20 & 57.1 & 40 & 56.3 \\
\hline \multicolumn{7}{|c|}{ Age group (years) } \\
\hline $16-25$ & 12 & 33.3 & 10 & 28.6 & 22 & 31 \\
\hline $26-35$ & 15 & 41.6 & 16 & 45.7 & 31 & 43.7 \\
\hline$>35$ & 9 & 25.1 & 9 & 25.7 & 18 & 25.3 \\
\hline
\end{tabular}

\section{Clinical efficacy of drugs under study}

A level of depression between two groups was comparable at the baseline. For amitriptyline group, decrease in mean HDRS score as compared to baseline at the end of two and four weeks was 6.14 and 13.50 respectively. Similarly for escitalopram group, mean decrease of 6.28 and 14.03 points was observed at the end of two and four week. The percentage reduction in the mean HDRS score for the amitriptyline group was $58.29 \%$ while that for the escitalopram group was $60.78 \%$. A change in mean HDRS score during the study period in amitriptyline and escitalopram group are summarized in Figure 1. When HDRS score at screening and four weeks were compared 
in both the groups, highly significant reduction in HDRS score was seen $(\mathrm{p}<0.05)$. Comparison of HDRS score between two groups at the end of study showed no significant difference $(\mathrm{p}>0.05)$.

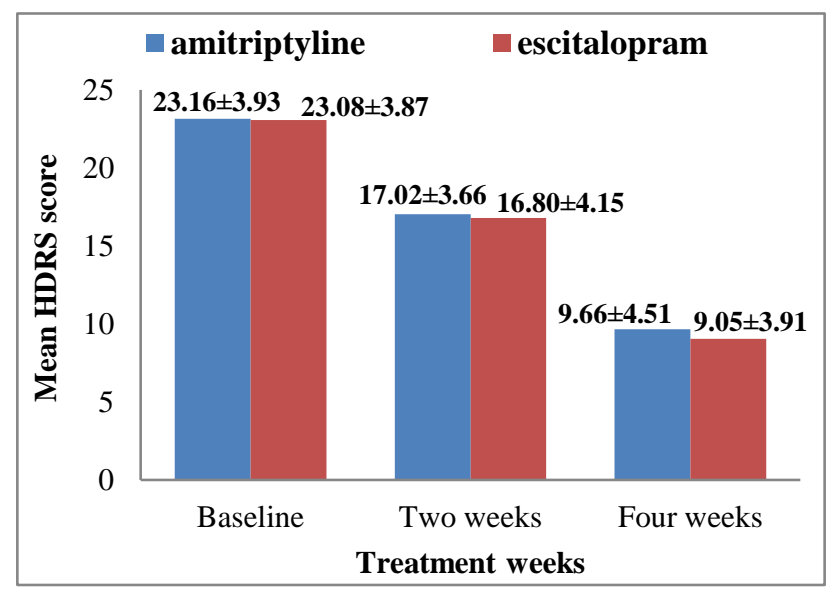

Figure 1: HDRS score during the study period in amitriptyline and escitalopram group.

\section{DISCUSSION}

In Nepal, data are limited regarding socio-demographic factor associated to depression. So, authors analyzed socio-demographical data although, due to methodological limitation, association of socio-demographic factors with depression could not be made with certainty. Our study revealed that depression is common in age group 16-35 years and the mean age of $32.84 \pm 12.35$ years (range 17-64 years) which were similar to previous studies. ${ }^{19,20}$ In contrast, other studies have found higher prevalence rate of depression in middle aged adults or in older age group. ${ }^{21,22}$ In the present study, prevalence of depression in females was higher which was comparable to previous studies. $^{20,21,23}$

In this study, a highly significant improvement in the HDRS score was observed in both the treatment groups at the end of four weeks. It indicated that amitriptyline and escitalopram are effective in the treatment of depression $(p<0.05)$ which is in accordance with numerous studies that have similar finding. $6,7,10,11$ When, HDRS score between amitriptyline and escitalopram, group were compared at the end of four weeks, no statistically significant difference was observed $(\mathrm{p}>0.05)$ which suggest equivalent efficacy between two groups. Our findings are comparable to several studies that have found TCAs and SSRIs have equal efficacy. ${ }^{13-16}$

Therefore, it can be concluded that amitriptyline and escitalopram are effective in the treatment of depression and the efficacy of escitalopram is equivalent to amitriptyline in Nepalese population.

Limitation of study was to the best knowledge, this study is the first to evaluate and compare efficacy of drug in the treatment of depression in Nepalese population, so more research is needed to confirm our finding. Also, this was open level, small scale four week study with the use of only one depression rating scale. In addition, this study did not assess tolerability profile of drug under study. Hence, authors recommend conducting a multicentre double-blind comparative study using multiple depression rating scale to assess both efficacy and tolerability of drug under study. Authors believe this study could be the basis for further studies in Nepal.

\section{ACKNOWLEDGEMENTS}

Authors would like to express their sincere gratitude to Prof. Dr. Vishnu Kant Kulshrestha for his exquisite guidance, suggestion, encouragement and inspiration. Authors would also like to thank all respondents for their participation.

Funding: No funding sources

Conflict of interest: None declared

Ethical approval: The study was approved by the Institutional Ethics Committee

\section{REFERENCES}

1. Kessler RC, Bromet EJ. The epidemiology of depression across cultures. Annu Rev Public Health. 2013;34:119-38.

2. Cassano P, Fava M. Depression and public health: an overview. J Psychosom Res. 2002;53(4):849-57.

3. Artigas F, Nutt DJ, Shelton R. Mechanism of action of antidepressants. Psychopharmacol Bull. 2002;36(2):123-32.

4. Palazidou E. Development of new antidepressants. Adv Psychiatr Treat. 1997;3:46-51.

5. Tatsumi M, Groshan K, Blakely RD, Richelson E. Pharmacological profile of antidepressants and related compounds at human monoamine transporters. Eur J Pharmacol. 1997;340:249-58.

6. Barbui C, Hotopf M. Amitriptyline vs the rest: still the leading antidepressant after 40 years of randomised controlled trials. Brit J Psychiat. 2001;178(2):129-44.

7. National Institute for Health and Clinical Excellence. Depression: the treatment and management of depression in adults. $2^{\text {nd }}$ Edition. Great Britain: RCPsych Publications; 2010:330.

8. Owens MJ, Knight DL, Nemeroff CB. Secondgeneration SSRIs: human monoamine transporter binding profile of escitalopram and R-fluoxetine. Biol Psychiat. 2001;50:345-50.

9. Jain R. Single-action versus dual-action antidepressants. Prim Care Companion J Clin Psychiatry. 2004;6(1):7-11.

10. Kennedy SH, Andersen HF, Lam RW. Efficacy of escitalopram in the treatment of major depressive disorder compared with conventional selective serotonin reuptake inhibitors and venlaxafine XR: a meta-analysis. J Psychiatry Neurosci. 2006;31(2):12231 . 
11. Culpepper L. Escitalopram. A New SSRI for the Treatment of Depression in Primary Care. Prim Care Companion J Clin Psychiatry. 2002;4(6):209-14.

12. Arroll B, Macgillivray S, Ogston S, Reid I, Sullivan F, Williams B, et al. Efficacy and tolerability of tricyclic antidepressants and SSRIs compared with placebo for treatment of depression in primary care: a metaanalysis. Ann Fam Med. 2005;3(5):449-56.

13. Von Wolff A, Holzel LP, Westphal A, Harter M, Kriston L. Selective serotonin reuptake inhibitors and tricyclic antidepressants in the acute treatment of chronic depression and dysthymia: a systematic review and meta-analysis. J Affect Disord. 2012;144(1-2):7-15.

14. Anderson IM, Tomerson BM. The efficacy of selective serotonin re-uptake inhibitors in depression: a meta-analysis of studies against tricyclic antidepressants. J Psychopharmacol. 1994;8:238-49.

15. Nierenberg AA. The treatment of severe depression: is there an efficacy gap between SSRI and TCA antidepressant generations? J Clin Psychiat. 1994;55:55-9.

16. Hirschfeld RM. Efficacy of SSRIs and newer antidepressants in severe depression: comparison with TCAs. J Clin Psychiat. 1999;60(5):326-35.

17. Olver JS, Burrows GD, Norman TR. Third-generation antidepressants: do they offer advantages over the SSRIs? CNS Drugs. 2001;15:941-54.
18. Barbey JT, Roose SP. SSRI safety in overdose. J Clin Psychiat. 1998;59(15):42-8.

19. Blay SL, Andreoli SB, Fillenbaum GG, Gastal FL. Depression morbidity in later life: prevalence and correlates in a developing country. Am J Geriat Psychiat. 2007;15:790-9.

20. Patten SB, Wang JL, Williams JV, Currie S, Beck CA, Maxwell CJ. Descriptive epidemiology of major depression in Canada. Can J Psychiat. 2006;51:84-90.

21. Hasin DS, Goodwin RD, Stinson FS, Grant BF. Epidemiology of major depressive disorder: results from the national epidemiologic survey on alcoholism and related conditions. Arch Gen Psychiat. 2005;62:1097-106.

22. Kolchakova PY, Akabaliev VH. A study of the effect of age on depressivity in Bulgarian urban population. Folia Med. 2003;45:11-5.

23. Aluoja A, Leinsalu M, Shlik J, Vasar V, Luuk K. Symptoms of depression in the Estonian population: prevalence, sociodemographic correlates and social adjustment. J Affect Disord. 2004;78:27-35.

Cite this article as: Aryal S, Chakrabarti K, Gupta M. A comparative study on efficacy of amitriptyline and escitalopram in the treatment of depression. Int $\mathrm{J}$ Basic Clin Pharmacol 2018;7:234-7. 